\title{
Development of Salt-sensing System for Jellyfish Desalting Process
}

\author{
Samak Rakmae, ${ }^{1 *}$ Pitikhate Sooraksa, ${ }^{2}$ and Pimpen Pornchaloempong ${ }^{1}$ \\ ${ }^{1}$ Department of Food Engineering, Faculty of Engineering, King Mongkut's Institute of Technology Ladkrabang, \\ 1 Chalongkrung, Ladkrabang, Bangkok 10520, Thailand \\ ${ }^{2}$ Department of Computer Engineering, Faculty of Engineering, \\ King Mongkut's Institute of Technology Ladkrabang, \\ 1 Chalongkrung, Ladkrabang, Bangkok 10520, Thailand
}

(Received Jun 6, 2019; accepted December 27, 2019)

Keywords: salinity measurement, desalting, seafood process, salt sensor, jellyfish

In the seafood industry, a large amount of salt is added to preserve seafood products. During processing, it is necessary to desalt the products for the sake of customers' health. Unlike a large-scale factory, many small enterprises lack tools and methods for desalting and measuring the efficiency of the desalting process. We have developed a rapid prototype salt-sensing system that can measure the desalting efficiency. Dried salted jellyfish are used as testing materials to evaluate the system. The rapid prototype comprises a microcontroller, a wire, and a liquid crystal display. Using a simple mapping between electrical conductivity and actual data obtained from the measurement of samples, the sensing system is successfully calibrated. A method of desalting the salted jellyfish material is also proposed. This desalting method and the newly developed simple sensing system for the desalting process are expected to make a significant contribution to the seafood processing industry.

\section{Introduction}

Cured jellyfish is a delicacy with high demand in Japan and other Asian countries. In the curing process, salt acts as a preservative by reducing the moisture content and maintaining microbial stability. ${ }^{(1)}$ The addition of salt is performed within a few hours of catching the jellyfish, while the animals are still alive. The salt content of salted jellyfish in Thailand is around $25-27 \%,{ }^{(2-4)}$ whereas it is around $16-25 \%{ }^{(2,4)}$ in some countries. The salting process generally takes around $20-40$ days and the final moisture content is around $60-70 \% .{ }^{(4)}$ Examples of processed jellyfish are shown in Fig. 1.

Reversely, the preparation of ready-to-use (RTU) products requires an overnight desalting procedure, which is a burden for busy individuals and even for modern industry. This problem may be overcome by adding sauces and flavors to the shredded RTU products. For example, for Japanese consumers, sliced RTU products are prepared with wasabi or mustard together with vinegar. ${ }^{(3)}$ However, the typical desalting process in industry of rinsing the jellyfish with fresh water until an acceptable salt content is reached is inefficient and time-consuming. In this

*Corresponding author: e-mail: samak.ra@kmitl.ac.th

https://doi.org/10.18494/SAM.2020.2463 


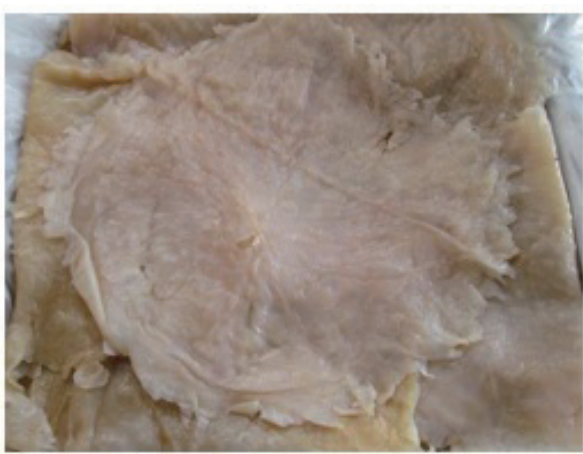

(a)

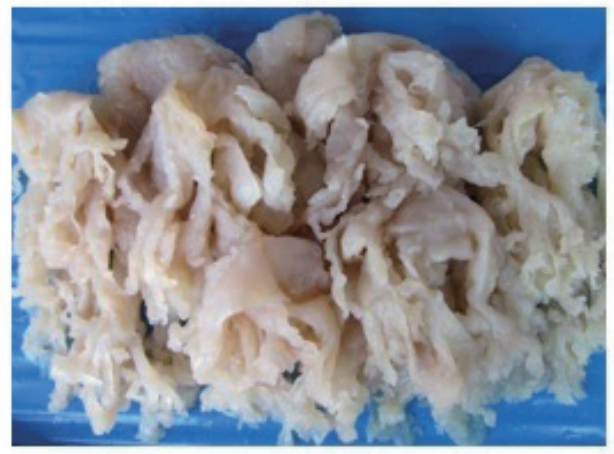

(b)

Fig. 1. (Color online) Processed jellyfish: (a) umbrella and (b) body.

paper, we propose an efficient and effective method for desalting cured jellyfish and report our newly developed salt-sensing system to detect the salt content of products.

This paper is organized as follows. In Sect. 2, we provide a brief review of the anatomy and physiology of jellyfish as a food material, the thermodynamics of desalting $\mathrm{NaCl}$, the development of a smart salt sensor, and the construction of a desalting machine suitable for small-scale enterprises. In Sect. 3, we present the results of our evaluation of the proposed system and a discussion. In Sect. 4, we give our conclusion.

\section{Materials and Methods}

\subsection{Anatomy}

Jellyfish are invertebrates with no brains. Around 17 species of jellyfish are edible, ${ }^{(5-9)}$ only three of which are consumed in Thailand: Rhopilema hispidum, Lobonema smithii, and Mastigias $s p{ }^{(7)}$ The animals come in a wide range of forms. Nevertheless, their bodies are similar. The body of an adult jellyfish, shown upside down in Fig. 2(a), comprises a bell-shaped body hood with an internal structure and an umbrella from which tentacles are suspended. As shown in Fig. 2(b), on the underside of the bell along the $x$-axis, four oral arms are connected to the manubrium in most species. The umbrella-shaped bell is a hollow structure consisting of a mass of transparent jellylike matter functioning as the hydrostatic skeleton of the animal, and $95 \%$ or more of these tissues consist of water. ${ }^{(9)}$ The high percentage of water contained in the cells implies that there is a water transport mechanism and corresponding optional conditions for water transport, which will be discussed in Sect. 2.2.

\subsection{Physiology}

According to previous research, ${ }^{(11)}$ there is evidence for aquaporin-mediated water transport in the jellyfish. This means that the water channels of cells can be used to transport water and regulate the volume of water in cells. In addition, further study by the same research group 


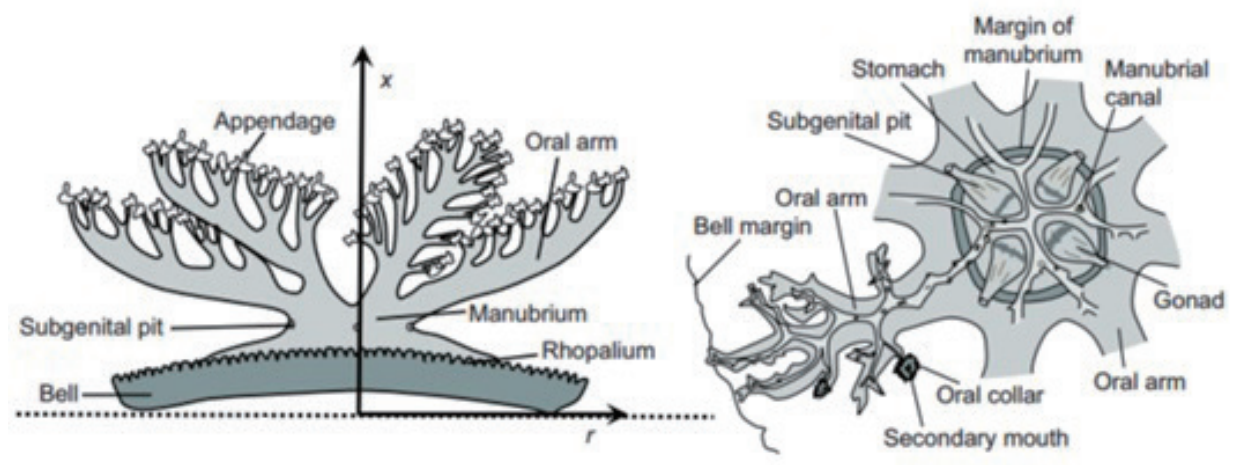

(a)

(b)

Fig. 2. (Color online) Anatomy of jellyfish: ${ }^{(10)}$ (a) side and (b) top views.

revealed that seawater acidification affects osmotic swelling, regulatory volume decrease (RVD), and discharge in the nematocysts of jellyfish. ${ }^{(12)}$ In an acidic environment, both the osmotic phase and the RVD are impaired. This implies that the alum (mixed with $\mathrm{NaCl}$ ) used for jellyfish preservation affects the water channels. This is due to the fact that when alum dissolves in water, it forms an acidic solution. In the design of a desalting process, it is necessary to reduce the proton concentration in the acid state caused by dissolved alum by adding a sufficient amount of fresh water to open the water channels of the cells as far as possible.

\subsection{Thermodynamics}

To ensure that the problem of jellyfish desalting is solvable, let us quickly examine whether the $\mathrm{NaCl}$ in the cells of dried salted jellyfish can dissolve in water when the jellyfish is placed in water. This is because we plan to use a conductive solution as an indicator of the salt quantity in water, which has an inverse relationship with the amount of salt remaining in the jellyfish. As known from thermodynamics, ${ }^{(13)}$ the dissolution of salt is an entropy-driven process. For a solution to form, the change in Gibbs free energy $(\Delta G)$ must be negative for dissolution. Recall that

$$
\Delta G=\Delta H-T \Delta S
$$

where $\Delta H$ is the change in enthalpy and $\Delta S$ is the change in entropy. By molecular simulation with a simple solubility model of $\mathrm{NaCl}$ in water, ${ }^{(14)}$ it has been revealed that $\Delta G$ is negative and has good agreement with the experimental value. This means that a salt-sensing system can be realized. The design and implementation of such a system are described in Sect. 2.4.

\subsection{Salt sensor}

Salinity is a measure of the amount of salt in water. The concept of the newly developed salt sensor is based on the fact that the salt that dissolves in water dissociates into negatively 
and positively charged ions. Conductivity is the ability of water to conduct an electrical current, where the dissolved ions are the conductors. The positively charged ions in salt water are sodium $\left(\mathrm{Na}^{+}\right)$and the negatively charged ions are chloride $\left(\mathrm{Cl}^{-}\right)$. If the temperature increases, the movement of ions also increases, and so does the conductivity. To compensate for temperature changes, the conductivity is commonly corrected to the value at a reference temperature. The measured conductivity is the sum of the conductivity from the water and that from the sodium and chloride ions. Because conductivity is a measure of the total concentration of ions, the method can be applied to indirectly monitor desalting performance. To develop a salt sensor, we employ this concept of solution conductivity measurement. Details are given as follows.

\subsubsection{Hardware}

Because we use deionized (DI) water in our system, the sample is noncorrosive and free of suspended solids. Hence, contact-based conductivity measurement with a two-electrode sensor is suitable in this case. Figure 3 shows a prototype of our rapid salt-sensing system. This sensor consists of two electrodes separated by a distance of $15 \mathrm{~mm}$ and attached to both sides of a tube, a temperature sensor (DS18D20), and an Arduino microcontroller. The sensing system has a simple structure designed for practical use. Similarly to the portable impedance-sensing system developed by Park et al., ${ }^{(15)}$ this system may be equipped with a transmitter and a Wi-Fi antenna to provide network connectivity by sending data to a smartphone. Unlike the impedancesensing system, this system uses an admittance sensor, namely, a conductance sensor.

\subsubsection{Mapping calibration}

No mapping algorithm to cope with temperature changes is perfect. Errors in the temperature measurement itself may lead to errors in the corrected conductivity. The conductivity can be increased if the temperature of an electrolyte solution increases. We use the

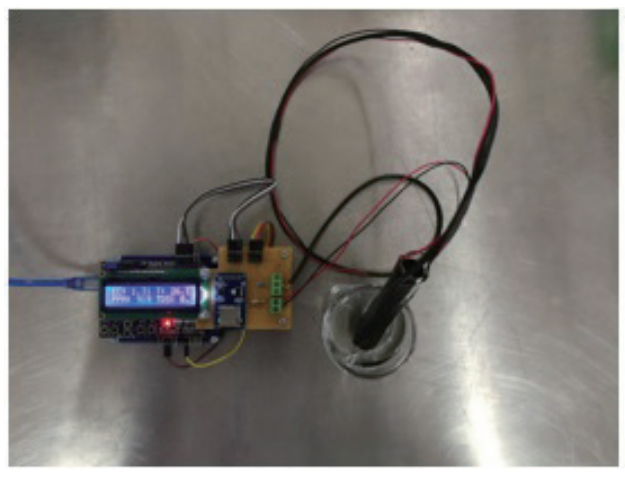

(a)

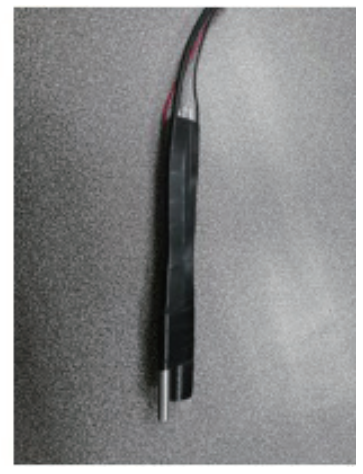

(b)

Fig. 3. (Color online) Salt-sensing system: (a) initial setup and (b) probe. 
temperature coefficient correction to correct this effect. The equation used ${ }^{(16)}$ is

$$
E C_{25}=E C(T)\left(1+\propto(T-25)^{-1}\right),
$$

where $E C(T)$ is the raw electrical conductivity at $T{ }^{\circ} \mathrm{C}, E C_{25}$ is the calculated conductivity at $25{ }^{\circ} \mathrm{C}$, and $\propto$ is the temperature coefficient, which approximately ranges from 1.8 to $3.0 \%$ for salt. Because $\propto$ is likely to be nonlinear with temperature, the accuracy can be improved if we limit the range of temperatures. We can use the following equation to calculate the linear temperature coefficient: ${ }^{(16)}$

$$
\propto=[E C(T 1)-E C(T 2)][(E C(T 1))(T 1-25)-(E C(T 2))(T 2-25)]^{-1},
$$

where $T 1$ and $T 2$ are two different solution temperatures.

\subsection{Desalting machine}

In Sect. 2.1, we have seen that the tissues of live jellyfish contain around $95 \%$ water, and in Sect. 2.2, we have seen that water reaches the cells via a water channel mechanism. Adding more water to dissolve the alum used to preserve the flavor and texture can help reduce the acidity or proton concentration. Eventually, this effect combined with the spontaneous dissolution of $\mathrm{NaCl}$ (Sect. 2.3) helps increase the osmotic pressure applied to the cells. In addition, the higher the temperature, the greater the solubility. We now state the design principle for our desalting machine, whose components are illustrated in Fig. 4.

1. The machine has a water tank for submerging dried salted jellyfish.

2. The machine has a temperature controller to control the water temperature.

3. The machine has a rotating mechanism to remove water from the jellyfish cells by centrifugation.

4. The machine is equipped with the salt-sensing unit in Sect. 2.4 to monitor the conductivity.

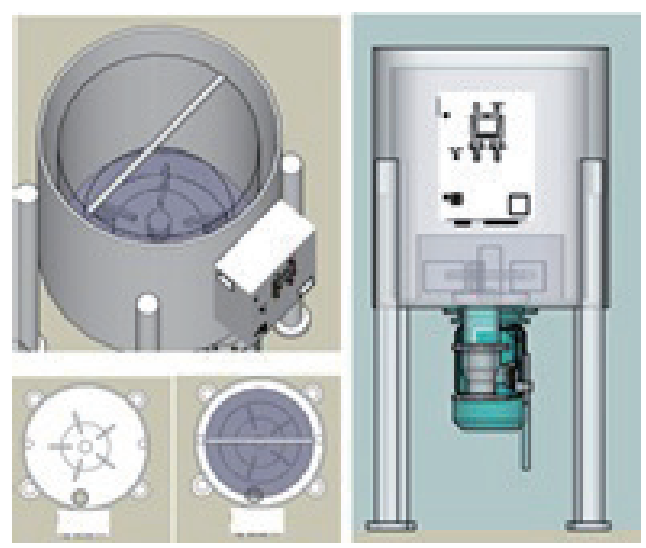

Fig. 4. (Color online) Conceptual drawing of the designed desalting machine. 


\section{Results and Discussion}

Three experiments were carried out and the results are presented in Sects. 3.1 and 3.2. In Sect. 3.1, we show the calibration of the values used to convert raw electrical signals into conductivity with temperature compensation. In Sect. 3.2, we demonstrate that such calibration is functionally effective.

\subsection{Evaluation of sensor accuracy}

In this subsection, we discuss the calibration of the values used to convert raw impedancelike signals into the calculated values of electrical conductivity $(\mu \mathrm{S} / \mathrm{cm})$ with temperature compensation referring to the electrical conductivity at $25{ }^{\circ} \mathrm{C}$. The standard references measured at $25{ }^{\circ} \mathrm{C}$ were DI water, distilled water, $5 \% \mathrm{NaCl}$, and $20 \% \mathrm{NaCl}$ prepared by weight. See Sect. 2.4 for calibration formulas and the mapping model. Figure 5 shows the experimental setup for the measurement and the results are shown in Table 1.

Table 1, Column 1 shows examples of raw values. The raw values of the samples are collected every $5 \mathrm{~s}$ and up to 2000 data points are collected. The values decrease from the top row to the bottom row, whereas the corresponding conductivities increase. This means that the input values are associated with impedance-like behaviors in the conversion process to the reciprocal admittance. Specifically, the procedure converts resistance into conductance. Columns 2 and 3 show the minimum and maximum values of the calculated electrical conductivity, respectively. Column 4 provides \%salt by weight in the test materials. Column 5 provides actual references based on measurement in the technical literature. ${ }^{(17,18)}$

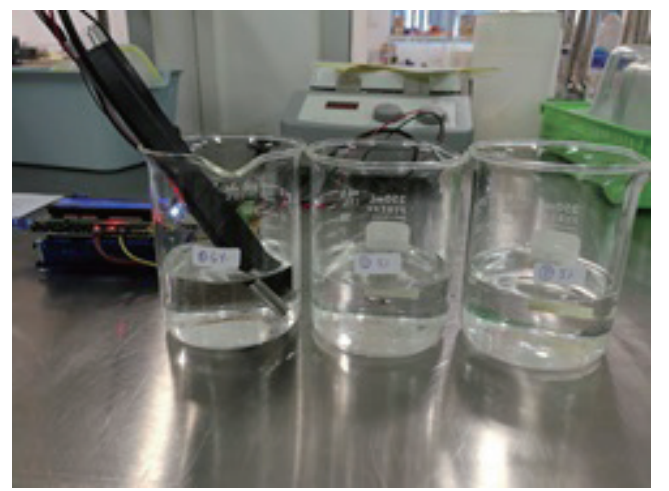

Fig. 5. (Color online) Calibration of electrical conductivity measurement versus references.

Table 1

Calculated electrical conductivities versus references in $\mu \mathrm{S} / \mathrm{cm}$ at $25^{\circ} \mathrm{C}$.

\begin{tabular}{cccccc}
\hline Examples of raw values & Min & Max & \% Salt by weight & Actual reference & Type of test sample \\
\hline 1048550 & 0.01 & 0.1 & 0 & $0.01-0.5^{(17)}$ & DI water \\
7150 & 1.4 & 4 & 0 & $1-5^{(17)}$ & Distilled water \\
702.25 & $36.99 \mathrm{k}$ & $38.43 \mathrm{k}$ & 5 & $75 \mathrm{k}^{(18)}$ & $5 \% \mathrm{NaCl}$ \\
391.76 & $226 \mathrm{k}$ & $247.1 \mathrm{k}$ & 20 & $23 \mathrm{k}^{(18)}$ & $20 \% \mathrm{NaCl}$ \\
\hline
\end{tabular}


Comparing the interval (Min, Max) in Columns 2 and 3 with that in Column 5, the calculated intervals between the minimum and maximum values contain the actual reference values in the literature. ${ }^{(17,18)}$ This means that the calculated values are valid because they fit both the order of magnitudes and the interval of the actual test sample. In addition, this suggests that the sensors can be employed for the measurement of salinity within an acceptable accuracy. Note that the maximum values of the conductivities of the DI water (deionized) and distilled water used in the test obtained from the conversion are 0.1 and $4 \mu \mathrm{S} / \mathrm{cm}$, respectively, and the $20 \% \mathrm{NaCl}$ solution yields a maximum conductivity of $2471000 \mu \mathrm{S} / \mathrm{cm}$.

When desalting jellyfish, the amount of $\mathrm{NaCl}$ by weight must be less than $1 \%$ of the product. Because dried salted jellyfish in the industry contains around $21 \% \mathrm{NaCl}$, we need to remove a salt content of at least $20 \%$. This means that the value of $2471000 \mu \mathrm{S} / \mathrm{cm}$ or $2.471 \mathrm{~S} / \mathrm{cm}$ measured from the solution in the desalting process can be set as a stopping criterion for each operation batch provided that the salt-sensing system can measure conductivity up to this value.

\subsection{Performance tests of the desalting machine}

The designed desalting machine built by the authors is shown in Fig. 6. The designed input target for the salted dried jellyfish is $50 \mathrm{~kg}$ for each batch. There are three $6 \mathrm{~kW}$ heaters with a temperature control unit installed in the system. A $2 \mathrm{hp} \mathrm{AC} \mathrm{motor} \mathrm{is} \mathrm{used} \mathrm{to} \mathrm{rotate} \mathrm{the} \mathrm{tank.} \mathrm{In}$ this performance test, the procedure begins with adding $150 \mathrm{~L}$ of water to half fill the container. We then load the jellyfish into the container and wash them for 1-2 min to remove the dirt. After draining the dirty water, we again fill the container with $150 \mathrm{~L}$ of water and set the control for the water temperature at $30-50{ }^{\circ} \mathrm{C}$ for $30 \mathrm{~min}$ to $3 \mathrm{~h}$ in an attempt to find the optimal temperature and time. We again drain the water from the container to complete the preparation process.

We now fill the container with $250-300 \mathrm{~L}$ of water to remove the $\mathrm{NaCl}$ from the jellyfish cells by spinning the container to apply osmotic pressure to the cells to let them absorb water. The spinning is stopped when the reading on the salt sensor is the value of the stopping criterion obtained from Sect. 3.1. We then take a sample of washed jellyfish and evaluate its moisture content and hardness. The results are shown in Figs. 7 and 8, respectively, where the horizontal

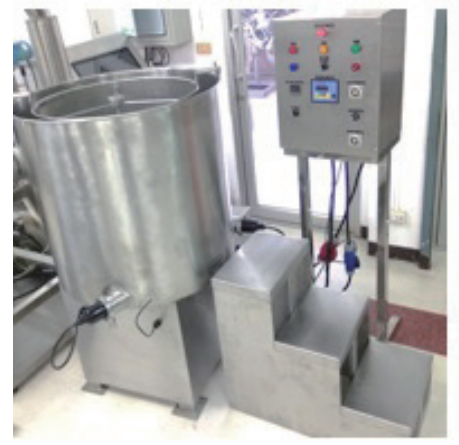

(a)

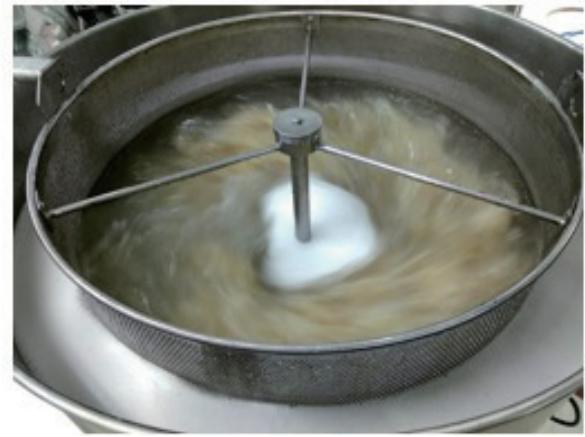

(b)

Fig. 6. (Color online) (a) Desalting machine. (b) Running in the centrifugal mode. 


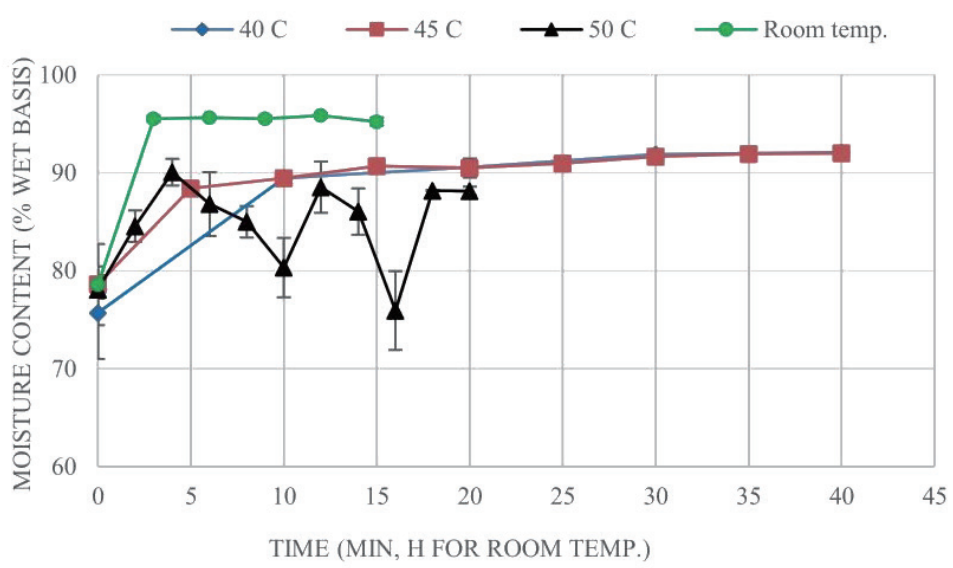

Fig. 7. (Color online) Moisture content of jellyfish over time for different processing temperatures.

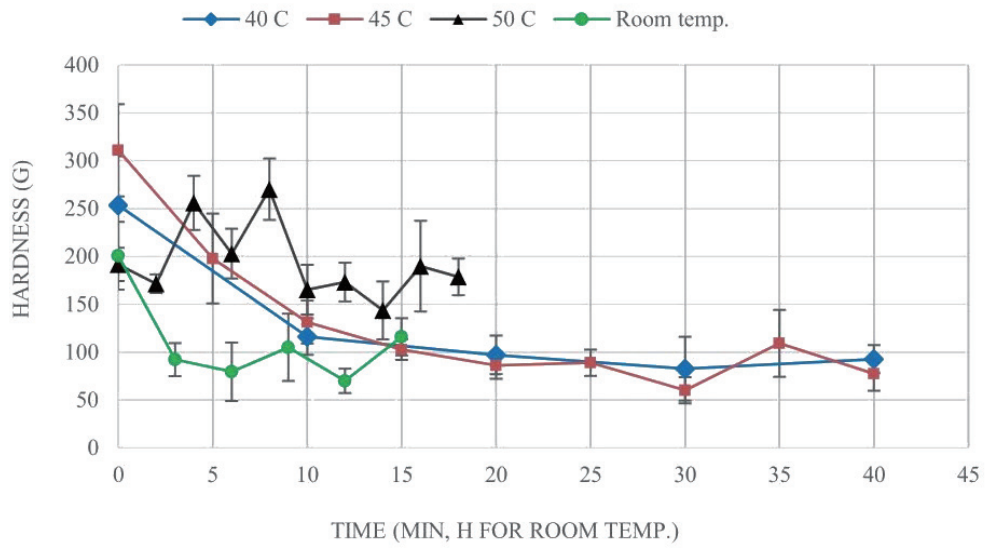

Fig. 8. (Color online) Results of maximum hardness test using the same conditions as in Fig. 7.

axis represents time (in min), except for the case of room temperature (in h), for which a longer time is required. As we can see from Fig. 7 , temperatures of $40{ }^{\circ} \mathrm{C}$ (diamonds) and $45^{\circ} \mathrm{C}$ (squares) during the submerging period yielded similar results, but that of $50{ }^{\circ} \mathrm{C}$ (triangles) produced unstable results. This indicates that at $50{ }^{\circ} \mathrm{C}$, the structure of the protein was changing. Hence, $40{ }^{\circ} \mathrm{C}$ is the optimal temperature for both satisfying the required moisture content and minimizing power consumption, while giving the closest results to those obtained at room temperature (circles). The results in Fig. 8 were obtained using a texture analyzer with a $3 \mathrm{~mm}$ pistol, where a puncture test was performed three times with six random samples at a speed of $2 \mathrm{~mm} / \mathrm{s}$. The force eventually settled at around $80-90 \mathrm{~g}$. Again, the sample at $50{ }^{\circ} \mathrm{C}$ did not have the required quality owing to protein deformation at this temperature. The temperature of $40{ }^{\circ} \mathrm{C}$ during submerging again appears to be optimal. Note that the data marks used in Fig. 8 are the same as those in Fig. 7. 


\section{Conclusion}

This study yielded two contributions: a method of desalting salted jellyfish and the development of a sensor system to detect salt dissolved in fresh water. The anatomy and physiology of jellyfish were discussed and analyzed to clarify the salt deposition mechanisms in the processed animals. The water channel mechanism gave us a concept for designing a centrifugal machine to remove $\mathrm{NaCl}$ molecules. According to the principles of thermodynamics, the change in Gibbs free energy for $\mathrm{NaCl}$ dissolved in water is negative, resulting in spontaneous dissolution. This means that $\mathrm{NaCl}$ is easily dissolved, even in roomtemperature water, and is easily removed from cells via the water channels.

Using this fact, we designed a simple yet very effective machine with a rotating water tank and temperature control. Finally, a simple smart portable device for salt measurement was developed and implemented. Mapping the conductance of the saline solution during the washing of jellyfish to the actual salt measurement in jellyfish provides an excellent stopping criterion for the desalting machine. Applying the findings would benefit the seafood processing industry by reducing the water consumption and time required for the desalting processes. This paper can also serve as a guideline for the development of salt-sensing systems and the desalting of other seafood products.

\section{Acknowledgments}

This study was supported in part by Thailand Research Funds (SRI5951203) and Center for Robotics and AI, King Mongkut's Institute of Technology Ladkrabang (KREF036205).

\section{References}

1 J. M. Kordylas: Processing and Preservation of Tropical and Subtropical Foods (Macmillan, London, 1990).

2 S. Subasinghe: Infofish Int. 4 (1992) 63.

3 Y.-H. P. Hsieh, F. M. Leong, and K. W. Barnes: J. Agric. Food Chem. 44 (1996) 3117. https://doi.org/10.1021/ jf950223m

4 Y.-H. P. Hsieh, F. M. Leong, and J. Rudloe: Hydrobiologia 451 (2001) 11. https://doi.org/10.1023/A:1011875720415

5 M. Kingsford, K. A. Pitt, and B. M. Gillanders: Oceanogr. Mar. Biol. 38 (2000) 85. https://www.researchgate. net/publication/285681656_Management_of_jellyfish_fisheries_with_special_reference_to_the_Order_ Rhizostomeae.

6 F. Muhammed and R. Sultana: Mar. Biodivers. Rec. 1 (2008) 1. https://doi.org/10.1017/S1755267207007294

7 M. Omori and E. Nakano: Hydrobiologia 451 (2001) 19. https://doi.org/10.1023/A:1011879821323

8 L. G. Suelo: PhD. Thesis, University of New South Wales, Sydney (1986).

9 Y.-H. Hsieh and J. Rudloe: Trends Food Sci. Technol. 5 (1994) 225. https://doi.org/10.1016/09242244(94)90253-4

10 A. Santhanakrishnan, M. Dollinger, C. L. Hamlet, S. P. Colin, and L. A. Miller: J. Exp. Biol. 215 (2012) 2369. https://doi.org/10.1242/jeb.053744

11 A. Marino, R. Morabito, G. La Spada, N. C. Adragna, and P. K. Lauf: Cell. Physiol. Biochem. 32 (2013) 77.

12 R. Morabito, A. Marino, P. K. Lauf, N. C. Adragna, and G. La Spada: Cell. Physiol. Biochem. 28 (2011) 1211.

13 R. A. Alberty: Biochemical Thermodynamics: Applications of Mathematica (Wiley, New Jersey, 2006).

14 E. Sanz and C. Vega: J. Chem. Phys. 126 (2007) 1. https://doi.org/10.1063/1.2397683

15 C.-Y. Park, J.-H. Min, Y.-S. Kim, H.-J. Song, and J.-D. Kim: Sens. Mater. 31 (2019) 253. https://doi. org/10.18494/SAM.2019.2091 
16 J. R. Gray, R. D. Down, and Y. H. Lehr: Environmental Instrumentation and Analysis Handbook (Wiley, New Jersey, 2004).

17 Emerson Process Management, Rosemount Analytical, Inc: Irvine, CA, USA. (2010) p. 1. https://www. emerson.com/documents/automation/application-data-sheet-theory-application-of-conductivity-rosemounten-68442.pdf

18 How to increase the accuracy of solution conductivity measurement (Online) p. 4. http://www.astisensor.com/ How_to_Increase_Accuracy_Conductivity_Measurements.pdf

\section{About the Authors}

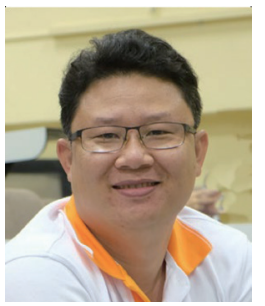

Samak Rakmae received his B.E. degree in food engineering from King Mongkut's Institute of Technology Ladkrabang (KMITL) in 2000 and his M.E. degree in food engineering from King Mongkut's University of Technology Thonburi (KMUTT), Thailand, in 2003. Since 2004, he has been a lecturer at KMITL. His research interests are in specific food machinery designs for small and medium enterprises (SMEs). He is currently a doctoral candidate at the Department of Food Engineering, Faculty of Engineering, KMITL. (samak.ra@kmitl.ac.th)

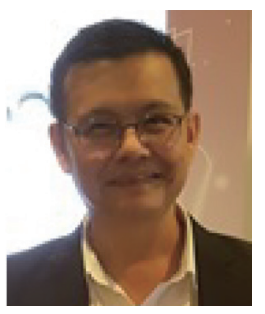

Pitikhate Sooraksa received his B.Ed. (Hons.) degree from Srinakharinwirot University, Patumwan, Thailand, in 1988 and his M.Sc. degree in electrophysics and his Ph.D. in electrical engineering from George Washington University, Washington DC, and the University of Houston, Texas, USA, in 1993 and 1996, respectively. From 1997 to the present, he has been working at KMITL, Thailand. His current research interests are in robotics and artificial intelligence and their applications.

(pitikhate.so@kmitl.ac.th)

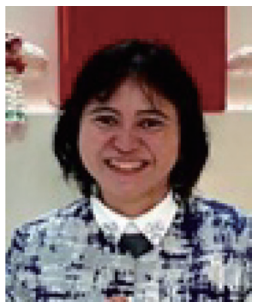

Pimpen Pornchaloempong received her B.S. and M.S degrees from Kasetsart University, Thailand, in 1987 and 1989, respectively, and her Ph.D. degree in food science from the Food Science and Human Nutrition University, Florida, USA, in 1999. Since 2010, she has been an assistant professor at KMITL, Thailand. Her research interests are in the thermal processing of food, the development of food processing, the effect of processing on food quality, and engineering properties of food. (pornchaloem.food@gmail.com) 